\title{
MATHEMATICAL MODEL FOR TISSUE STRESSES IN GROWING PLANT CELLS AND ORGANS
}

\author{
SYlWia LEWICKA, MARIUSZ PIETRUSZKA \\ Department of Plant Physiology, \\ Faculty of Biology and Environmental Protection, University of Silesia \\ Jagiellońska 28, 40-032 Katowice, Poland \\ e-mail: slewicka@us.edu.pl
}

(Received: October 5, 2007. Accepted: April 2, 2008)

\begin{abstract}
In this study we propose a simple mathematical model based on the equilibrium equation for the materials deformed elastically. Owing to the turgor pressure of the cells, the peripheral walls of the outer tissue are under tension, while the extensible inner tissue is under compression. This well known properties of growing multicellular plant organs can be derived from the equation for equilibrium. The analytic solutions may serve as a good starting point for modeling the growth of a single plant cell or an organ.
\end{abstract}

KEY WORDS: equilibrium equation, growing plant cell/organ, tissue stresses.

\section{INTRODUCTION}

The notion of growth tensors in developing plant organs has been known for a long time (see Kutschera 1989 for a review). The mathematical description for such systems has been successfully applied to apical meristems where the proliferating cells produce tissue stresses. The newly formed stresses influence the structure of the growing organ and, in particular, the principal directions of growth; also enforces the natural coordinate system which manage the further development of the organ (Hejnowicz et al. 1984; Hejnowicz 1984). A different situation one observes for elongating plant organs, such as coleoptiles in young grasses or elongation zones in roots, where cells division takes place very rarely. Various stresses occur because of different properties of cell walls in the organ growing due to the water uptake driven by the gradient in water potential, Kutschera 2000. In particular, walls in the outer tissues are more rigid and thick, while inner tissues are elastic and thin walled. Thus, in an elongating plant organ, the outer tissue in under tension and the inner one - under compression (Kutschera 1989, 1995; Hejnowicz and Sievers 1995). The distribution of the tissue stresses as well as deformation of the particular tissue layers can be found using one of the equilibrium equations for deformed materials. In theory of elasticity, among commonly used constitutive equations (describing mathematical relations between stress and strain, or movement of a body), equations for equilibrium play a significant role in solving the problem of finding the deformation of the bulk which undergoes an external action (Landau and Lifszyc 1993; Atkin and Fox 2005). The equilibrium equation may be derived for materials deformed elastically or non-elastically. In the first case, the displacement vector $\vec{u}$ is time-independent, in contrary to the second case. In this article we focus on the elastic properties in order to solve the equation using analytic methods. Such approach may be considered as a first step towards further development of the model where the numerical calculations may be unavoidable.

\section{MODEL}

Assuming no external volumetric force (like gravity) acting on the body, the equilibrium equation for the displacement vector $\vec{u}$ takes on the form

$$
2(1-v) \nabla(\nabla \cdot \vec{u})-(1-2 v) \nabla \times(\nabla \times \vec{u})=\overrightarrow{0},
$$

where $\nabla$ (nabla) is a differential operator, $v$ - Poisson coefficient. Eq. (1) can be analytically solved when the problem exhibits a high degree of symmetry, i.e. cylindrical or spherical one. A biological argument leads us to consider the cylindrical symmetry, since elongating cells or organs often have such a shape (e.g. internode cell of algae Nitellopsis obtusa L., or coleoptiles of Graminae). Now, we wish to find an appropriate model reflecting elongation of (a) a single plant cell and (b) plant organ. 
In cylindrical coordinate system vector operators grad, div and curl become

$$
\begin{aligned}
& \operatorname{grad} A=\frac{\partial A}{\partial r} \hat{e}_{r}+\frac{1}{r} \frac{\partial A}{\partial \phi} \hat{e}_{\phi}+\frac{\partial A}{\partial z} \hat{e}_{z} \\
& \operatorname{div} \vec{V}=\frac{1}{r} \frac{\partial}{\partial r}\left(r V_{r}\right)+\frac{1}{r} \frac{\partial V_{\phi}}{\partial \phi}+\frac{\partial V_{z}}{\partial z} \\
& \operatorname{curl} \vec{V}=\left(\frac{1}{r} \frac{\partial V_{z}}{\partial \phi}-\frac{\partial V_{\phi}}{\partial z}\right) \hat{e}_{r}+\left(\frac{\partial V_{r}}{\partial z}-\frac{\partial V_{z}}{\partial r}\right) \hat{e}_{\phi}+\frac{1}{r}\left(\frac{\partial}{\partial r}\left(r V_{\phi}\right)-\frac{\partial V_{r}}{\partial \phi}\right) \hat{e}_{z}
\end{aligned}
$$

(true for arbitrary scalar or vector fields $A$ and $\vec{V}$, respectively) and the problem significantly complicates. However, if we assume that the displacement field

$$
\vec{u}=\left(u_{r}, u_{\phi}, u_{z}\right)=\left(u_{r}, 0,0\right)
$$

has magnitude depending only on the distance $r$, then Eq. (1) becomes (through Eq. (2))

$$
\begin{gathered}
\frac{\mathrm{d}}{\mathrm{d} r}\left(\frac{1}{r} \frac{\mathrm{d}}{\mathrm{d} r}\left(r u_{r}\right)\right)=0 \\
\Downarrow \\
u_{r}=a r+\frac{b}{r}
\end{gathered}
$$

Here we propose a model for an elongating plant cell as a hollow cylinder which can be thin or thick (in analogy to thin or thick cell wall, respectively). The cylinder is immersed in a medium (with pressure $p_{a}$ ), and is filled by a homogeneous fluid which plays a role of cell sap (with pressure $P-Y$, where $Y$ - yield threshold). Elastic properties of the cylinder (cell wall) are represented by two physical quantities: Young's modulus $\varepsilon$ and Poisson coefficient $v$.

For an elongating plant organ, in turn, it seems that a filled cylinder (elastic core) covered by a thin layer of quite rigid material can be a good analytic model. The core is an analogue to the inner tissue of the organ, while the layer is an equivalent of the outer tissue. In elongating organs, the inner tissue is an assembly of cells with thin elastic walls, and the outer tissue is composed mainly of epidermal cells which have thick and rigid walls. In our model, these properties are reflected by different Young's moduli and Poisson coefficients $\left(\varepsilon_{1}, v_{1}\right.$ for the core, $\varepsilon_{2}, v_{2}$ for the overlay). The question is whether such simple models can reflect stresses in the cell wall in (a) a single plant cell or apoplast in (b) plant organ during growth. This interesting problem is discussed in the last section.

\section{SOLUTIONS}

For the model of an elongating plant cell the solution is $u_{r}(r)=a r+b / r$ with two parameters $a$ and $b$ determined from the boundary conditions, which are expressed for the stress tensor $\{\sigma\}_{i j}$ :

- $\sigma_{r r}=-p_{a}$ for $r=\mathrm{R}_{i}(i=1,2$ depending on whether we are dealing with thin or thick cylinder, $\left.\mathrm{R}_{2}>\mathrm{R}_{1}\right)$

- $\sigma_{r r}=-(P-Y)$ for $r=\mathrm{R}_{0}$ (the inner radius is equal in both cases),

see Fig. 1. Now, because in considered models we take into account only relatively small elastic deformations, the stress $\{\sigma\}_{i j}$ and strain $\{u\}_{i j}$ tensors are related by the Hooke's law. Beneath we present only the expression for $\sigma_{r r}$

$$
\sigma_{r r}=\frac{\varepsilon}{(1+v)(1-2 v)}\left((1-v) u_{r r}+v\left(u_{\phi \phi}+u_{z z}\right)\right)
$$

Because of the high degree of symmetry $\vec{u}=\left(u_{r}(r), 0,0\right)$ and the diagonal elements of the strain tensor are

$$
u_{r r}=\frac{\mathrm{d} u_{r}}{\mathrm{~d} r}=a-\frac{b}{r^{2}}, u_{\phi \phi}=\frac{u_{r}}{r}=a+\frac{b}{r^{2}}, u_{z z}=0 .
$$

Furthermore, all off-diagonal elements vanish. Then, the $r r$ and $\phi \phi$ elements of the stress tensor $\{\sigma\}_{i j}$ have the form as follows

$$
\begin{aligned}
\sigma_{r r} & =\frac{\varepsilon}{(1+v)(1-2 v)}\left(a-(1-2 v) \frac{b}{r^{2}}\right) \\
\sigma_{\phi \phi} & =\frac{\varepsilon}{(1+v)(1-2 v)}\left(a+(1-2 v) \frac{b}{r^{2}}\right) .
\end{aligned}
$$

These equations have been used to determine the parameters $a$ and $b$, or - in the second model of plant organ $a_{1}, a_{2}$ and $b_{2}$. After some calculations one can obtain the following expressions for $a$ and $b$ :

\section{MODELS OF A SINGLE CELL}

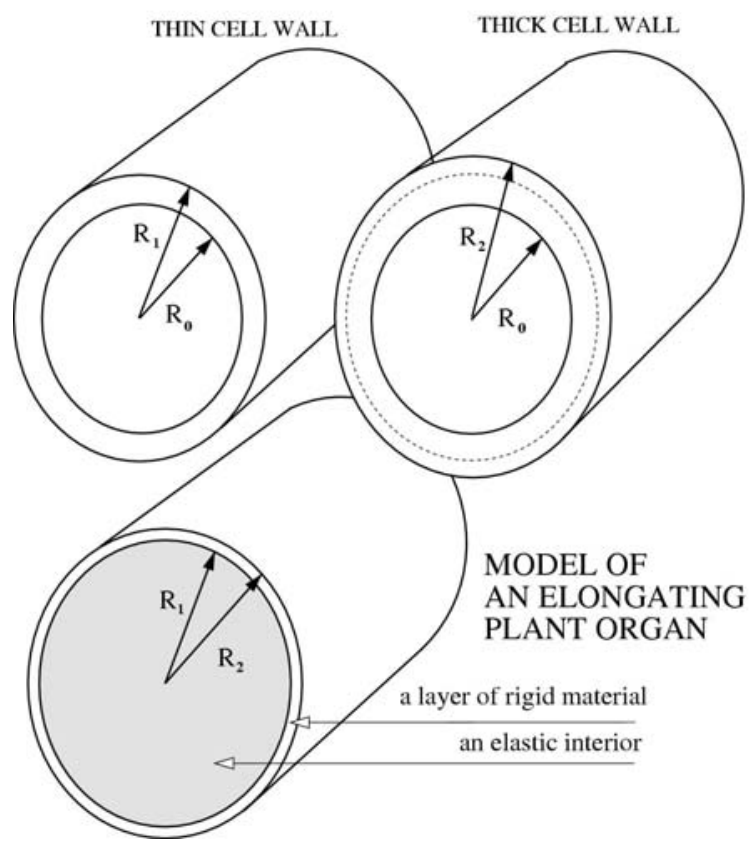

Fig. 1. Models for elongating (a) plant cell (with thin or thick elastic cell wall) and (b) plant organ: elastic interior (Poisson coefficient $v_{1}<0.5$, Young's modulus $\left.\varepsilon_{1} \sim 0.1\right)$ covered by a rigid layer $\left(v_{2}<<v_{1}, \varepsilon_{2} \sim 1\right)$. 
(7)

$$
\begin{aligned}
& a=\frac{(1+v)(1-2 v)}{\varepsilon\left(\mathrm{R}_{i}^{2}-\mathrm{R}_{0}^{2}\right)}\left[(P-Y) \mathrm{R}_{0}^{2}-p_{a} \mathrm{R}_{1}^{2}\right] \\
& b=\frac{1+v}{\varepsilon}\left(P-Y-p_{a}\right) \frac{\mathrm{R}_{0}^{2} \mathrm{R}_{1}^{2}}{\mathrm{R}_{1}^{2}-\mathrm{R}_{0}^{2}}
\end{aligned}
$$

For the model of an elongating plant organ the solution is more complicated:

$$
\text { (8) } \vec{u}=\left\{\begin{array}{cc}
\vec{u}_{1}=\left(a_{1} r, 0,0\right) & \text { for } r \leq R_{1} \text { (within the core) } \\
\vec{u}_{2}=\left(a_{2} r+b_{2} / r, 0,0\right) & \text { for } R_{1} \leq r \leq R_{2} \text { (in the outlay) }
\end{array}\right.
$$

and has three parameters which can be found from a single boundary condition and two continuity conditions:

$$
\begin{aligned}
& \text { - } \sigma_{r r}=p \text { for } r=\mathrm{R}_{2} \\
& \text { - } \lim _{r \rightarrow \mathrm{R}_{1}^{+}} u_{r}=\lim _{r \rightarrow \mathrm{R}_{1}^{-}} u_{r} \Leftrightarrow u_{1}\left(r=\mathrm{R}_{1}\right)=u_{2}\left(r=\mathrm{R}_{1}\right) \\
& \text { - } \lim _{r \rightarrow \mathrm{R}_{1}^{+}} \sigma_{r r}=\lim _{r \rightarrow \mathrm{R}_{1}^{-}} \sigma_{r r} \Leftrightarrow \sigma_{r r}^{(1)}\left(r=\mathrm{R}_{1}\right)=\sigma_{r r}^{(2)}\left(r=\mathrm{R}_{1}\right)
\end{aligned}
$$

The latter two conditions need a comment. The assumption that the displacement vector has to be continuous is natural - during growth the organ shouldn't tear. Radial element of the stress tensor $\sigma_{r r}$ should also be continuous, because the discontinuity would affect the appearance of some force acting on the surface $r=\mathrm{R}_{1}$ causing attraction or repulsion between the core and the outlay. We believe that such situation is not the case here - in the growing organ such dislocations and additional pressure in the interface do not occur. The above listed conditions generate expressions for $a_{1}, a_{2}$ and $b_{2}$ coefficients and can be found in the Appendix.

\section{RESULTS}

In both cases, (a) and (b), we have calculated radial coordinate $u_{r}(r)$ of the displacement vector, also radial $\sigma_{r r}$ and angular $\sigma_{\phi \phi}$ elements of the stress tensor $\{\sigma\}_{i j}$, both dependent only on the radial coordinate $r$. We put the structural $\mathrm{R}_{0}, \mathrm{R}_{1}, \mathrm{R}_{2}$, material parameters $\mathrm{v}, \varepsilon, v_{1}, v_{2}, \varepsilon_{1}, \varepsilon_{2}$ and medium parameters $p_{a}, p, P-Y$, as follows:

(a) $\mathrm{R}_{0}=1, \mathrm{R}_{1}=1.375, \mathrm{R}_{2}=1.5, v=0.1$ and $\varepsilon=1$ (quite elastic material)

(b) $\mathrm{R}_{1}=2$ and $\mathrm{R}_{2}=2.25, v_{1}=0.4$ and $\varepsilon_{1}=0.1$ (very elastic material - physically similar to rubber) and $v_{2}=0.01$ and $\varepsilon_{1}=3$ (quite rigid material). In all cases the radius is given in $[\mu \mathrm{m}]$, the Young's modulus in $\left[10^{3} \mathrm{MPa}\right]$ and the Poison coefficient - dimensionless.

The pressure $P-Y$ has been given as $0.3 \mathrm{MPa}$ (as in typical elongating plant cell), $p_{a}=0.1 \mathrm{MPa}$ and $p=0.2 \mathrm{MPa}$.

In Fig. 1 we have visualized both models for elongating (a) plant cell and (b) organ. The main conclusion from the results presented in Figs 2-7 is drastic lowering of the deformation when thickening the cylinder's wall in case (a) or - when covering the elastic core by a rigid layer in (b). The radial and angular elements of the stress tensor, $\sigma_{r r}$ and $\sigma_{\phi \phi}$, have been presented in Figs 5-6, respectively. In Fig. 7 one can see that the presence of the outer layer causes decrease of $\sigma_{r r}$ within the whole cylinder, however, in the elastic core $\sigma_{r r}$ remains constant, while in the outlay it increases up to $p$ (left box). Different response has been obtained for the angular element $\sigma_{\phi \phi}$, namely an abrupt discontinuity
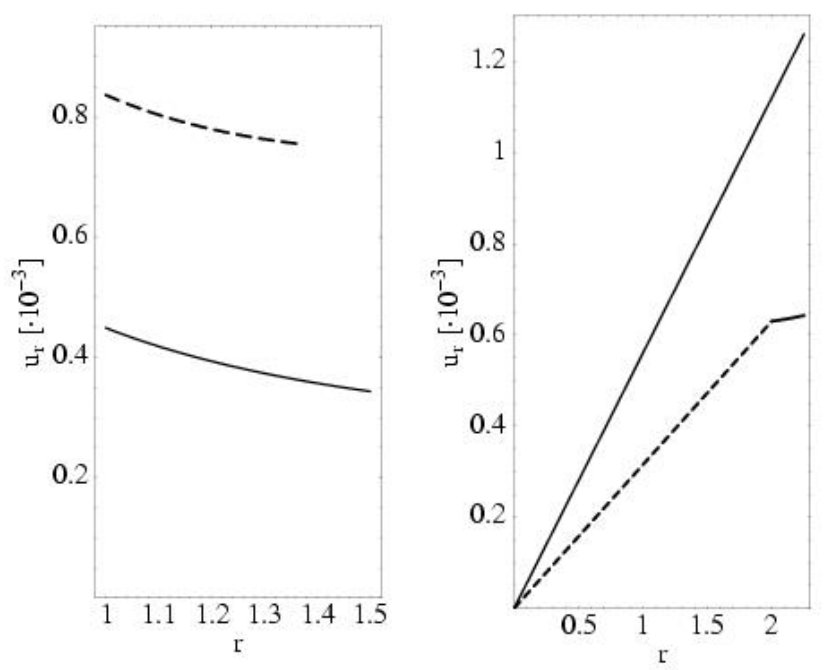

Fig. 2. Radial coordinate $u_{r}(r)$ of the displacement vector $\vec{u}=\left(u_{r}, 0,0\right)$ obtained via theoretical calculations from the equilibrium equation. The left figure presents $u_{r}$ dependence on $r$ in two cases of a hollow thin (dashed line) or thick cylinder (solid line). The right figure presents $u_{r}(r)$ in the filled elastic cylinder covered by a thin layer of rigid material (the model of an elongating organ). As a reference a plot of "bare" elastic organ has been added (solid line).

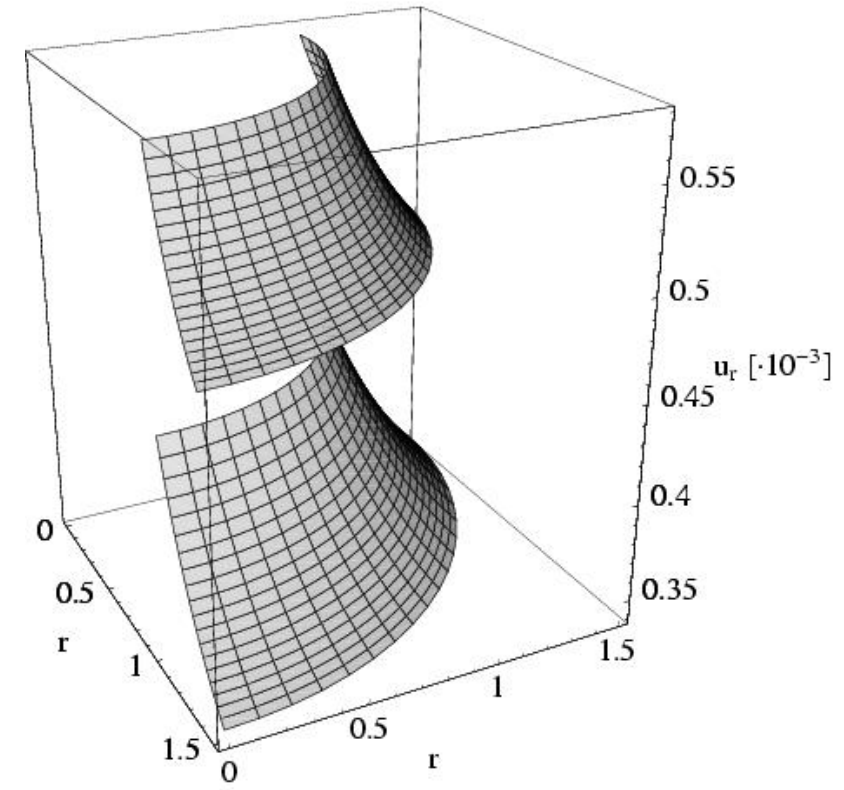

Fig. 3. Visualization (in a form of parametric 3D plot obtained for angles $0 \leq \phi \leq \pi / 2)$ of the single cell analytic model. The figure presents the radial coordinate of the displacement vector $\vec{u}$; thin cell wall - upper plot, thick cell wall - lower plot.

at $r=\mathrm{R}_{1}$ occurs. In the elastic core $\sigma_{\phi \phi}$ is lowered with respect to the uncovered "bare" core (indicated in Fig. 7 by the solid line), but in the outlay $\sigma_{\phi \phi}$ is much greater (right box). Both results presented in Fig. 7 we may refer to the compression in the inner tissue and tension in the outer tissue (see Kutschera 1989, 2000).

\section{DISCUSSION}

The aim of the present study was to find the distribution of the tissue stresses and deformation of elongating plant cells and organs using simple mathematical formulae. The equilibrium equation for deformed bodies is undoubtedly 


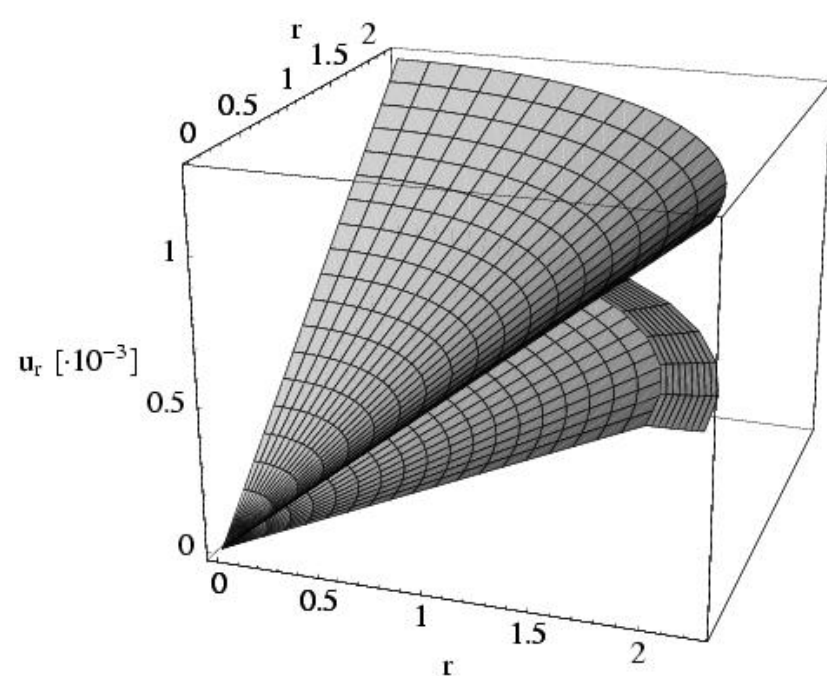

Fig. 4. Visualization (in a form of parametric 3D plot obtained for angles $0 \leq \phi \leq \pi / 2)$ of the elongating organ model. The figure presents the radial coordinate $u_{r}$; homogeneous "bare" elastic material - upper plot, elastic material covered by a thin rigid layer - lower plot.

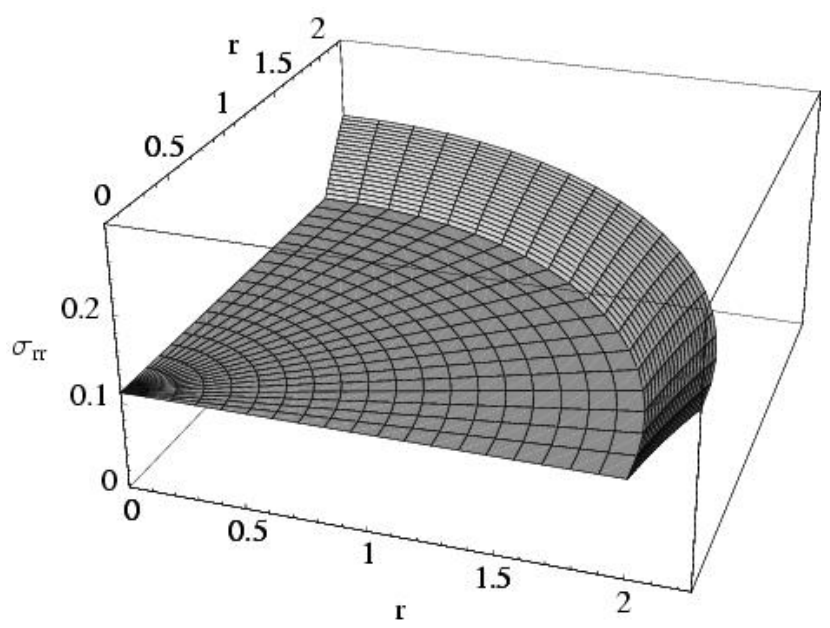

Fig. 5. The diagonal $r$ element of the stress tensor $\{\sigma\}_{i j}$ as a function of radius $r$ and angle $\phi-3 \mathrm{D}$ visualization of the model for an elongating organ.

a good candidate, as it has been derived from principle laws (energy conservation law and continuity equation) and as such is universal. In systems with high degree of symmetry, the equation (for bodies deformed elastically) can be solved analytically, and solutions are easy to interpret. Nonetheless, the question of its appropriateness still remains. For an elongating plant cell, the solution is exact, if we do not take into account other shapes of the cell than cylindrical, and treat the cell wall as a homogeneous structure. However, an elongating organ is a multicellular organism, so the model is satisfactory, if we are interesting in general and qualitative description. Such description is also simplified as we divide the organ only into two compartments: outer and inner tissue. Both are homogeneous, but possess different mechanical properties. Further development should account for the multicellularity, i.e. the more precise model should consider more complicated boundary conditions. Especially, the elements of the deformation and stress tensors depend on all spatial coordinates: $r, \phi$, and $z$.

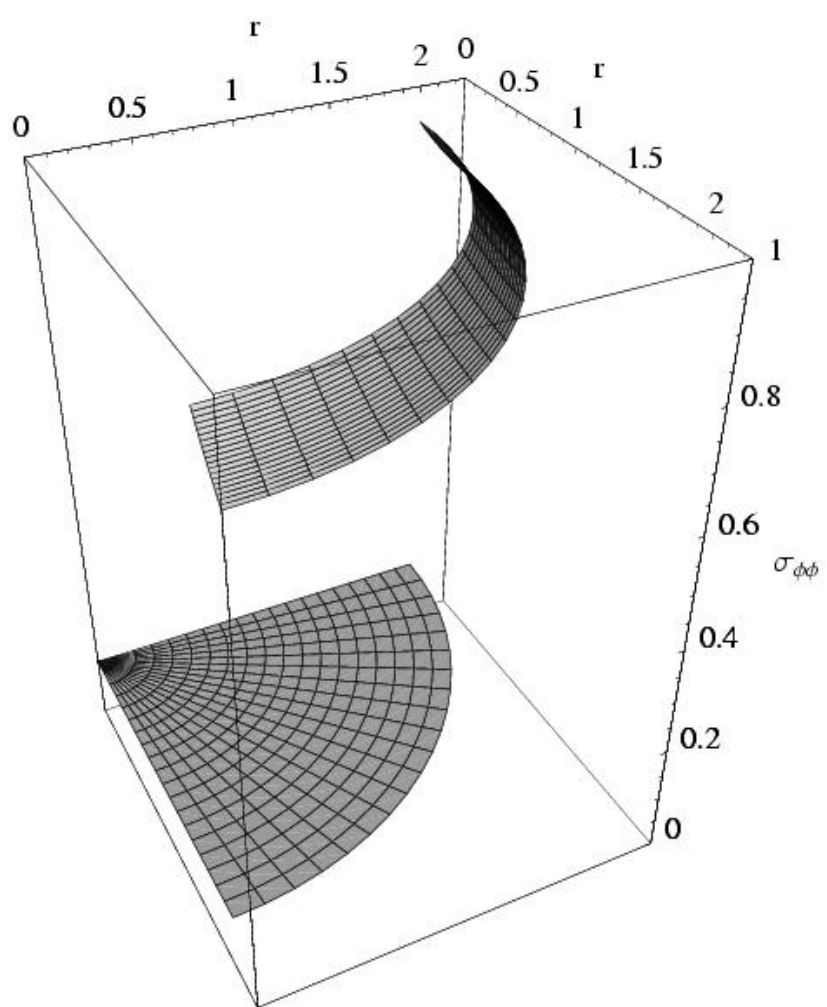

Fig. 6. The diagonal $\phi \phi$ element of the stress tensor $\{\sigma\}_{i j}$ as a function of radius $r$ and angle $\phi-3 \mathrm{D}$ visualization of the model for an elongating organ.
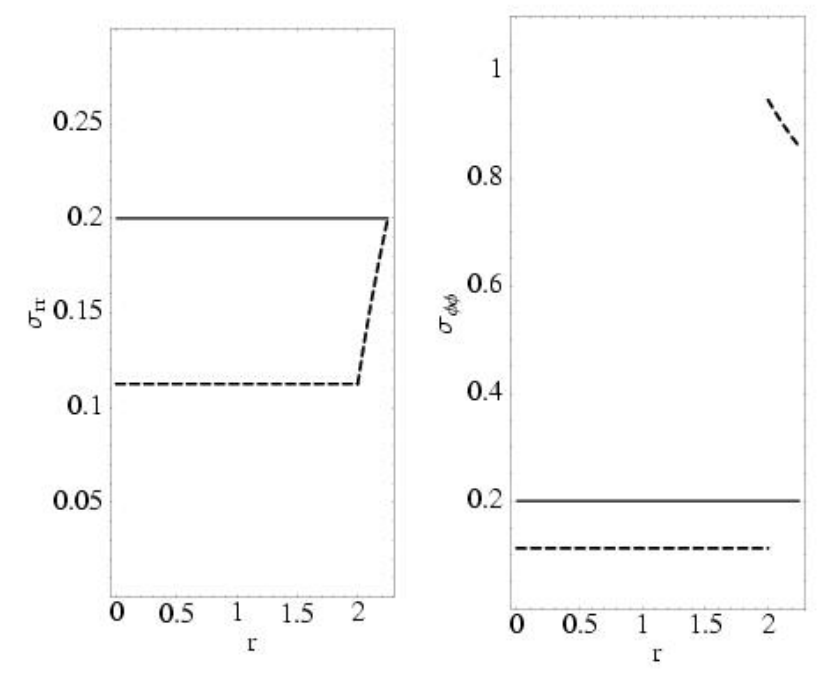

Fig. 7. Plot of the calculated $\sigma_{r}$ and $\sigma_{\phi \phi}$ versus radius $r$ for the covered cylinder. The solid line represents the pressure within the "bare" cylinder.

Moreover, wishing to find both elastic and plastic properties of the growing organ one should use the equilibrium equation for bodies deformed visco-elastically. It means that since vector $\vec{u}$ depends also on time, the problem complicates and the system may need to be solved using numerical methods.

\section{APPENDIX}

After some tedious calculations the expressions for $a_{1}, a_{2}$ and $b_{2}$ read: 
(9)

$$
\begin{aligned}
& a_{1}=\frac{p}{\varepsilon_{2}}\left(1+v_{2}\right)\left(1-2 v_{2}\right)+\left(1-\frac{\varepsilon_{1}}{\varepsilon_{2}} \frac{\left(1+v_{2}\right)\left(1-2 v_{2}\right)}{\left(1+v_{1}\right)\left(1-2 v_{1}\right)}\right) \frac{p\left[\left(1-2 v_{2}\right) R_{1}^{2}+R_{2}^{2}\right]}{\frac{\varepsilon_{1}}{\left(1+v_{1}\right)\left(1-2 v_{1}\right)}\left(\left(1-2 v_{2}\right) R_{1}^{2}+R_{2}^{2}\right)+\frac{\varepsilon_{2}}{\left(1+v_{2}\right)}\left(R_{2}^{2}-R_{1}^{2}\right)} \\
& a_{2}=\frac{p}{\varepsilon_{2}}\left(1+v_{2}\right)\left(1-2 v_{2}\right)+\left(1-\frac{\varepsilon_{1}}{\varepsilon_{2}} \frac{\left(1+v_{2}\right)\left(1-2 v_{2}\right)}{\left(1+v_{1}\right)\left(1-2 v_{1}\right)}\right) \frac{\left(1-2 v_{2}\right) p R_{1}^{2}}{\left.\frac{\varepsilon_{1}}{\left(1+v_{1}\right)\left(1-2 v_{1}\right)}\left(1-2 v_{2}\right) R_{1}^{2}+R_{2}^{2}\right)+\frac{\varepsilon_{2}}{\left(1+v_{2}\right)}\left(R_{2}^{2}-R_{1}^{2}\right)} \\
& \left.b_{2}=\left(1-\frac{\varepsilon_{1}}{\varepsilon_{2}} \frac{\left(1+v_{2}\right)\left(1-2 v_{2}\right)}{\left(1+v_{1}\right)\left(1-2 v_{1}\right)}\right) \frac{\varepsilon_{1}}{\left(1+v_{1}\right)\left(1-2 v_{1}\right)}\left(1-2 v_{2}\right) R_{1}^{2}+R_{2}^{2}\right)+\frac{\varepsilon_{2}}{\left(1+v_{2}\right)}\left(R_{2}^{2}-R_{1}^{2}\right)
\end{aligned}
$$

\section{LITERATURE CITED}

ATKIN R.J., FOX N. 2005. An introduction to the theory of elasticity. Dover Publications Inc., New York.

HEJNOWICZ Z., NAKIELSKI J., HEJNOWICZ K. 1984. Modeling of spatial variations of growth within apical domes by means of the growth tensor. I. Growth specified on dome axis. Acta Soc. Bot. Pol. 53: 17-28.

HEJNOWICZ Z. 1984. Trajectories of principal directions of growth, natural coordinate system in growing plant organ. Acta Soc. Bot. Pol. 53: 29-42.

HEJNOWICZ Z., SIEVERS A. 1995. Tissue stresses in organs of herbaceous plants. I. Poisson ratios of tissues and their role in determination of the stresses. J. Exp. Bot. 46: 1035-1043.
HEJNOWICZ Z. 1997. Graviresponses in herbs and trees: a major role for the redistribution of tissue and growth stresses. Planta 203: S136-S146.

KUTSCHERA U. 1989. Tissue stresses in growing plant organs. Physiol. Plant. 77: 157-163.

KUTSCHERA U. 1995. Tissue pressure and cell turgor in axial plant organs: implications for the organismal theory of multicellularity. J. Plant Physiol. 146: 126-132.

KUTSCHERA U. 2000. Cell expansion in plant development. R. Bras. Fisiol. Veg. 12: 65-95.

LANDAU L.D., LIFSZYC E.M. 1993. Teoria sprężystości. Wydawnictwo Naukowe PWN, Warszawa. 